EUROPEAN ORGANIZATION FOR NUCLEAR RESEARCH

CERN-EP/2001-082

November 16th, 2001

\title{
Search for Doubly Charged Higgs Bosons with the OPAL detector at LEP
}

\author{
The OPAL Collaboration
}

\begin{abstract}
A search for pair-produced doubly charged Higgs bosons has been performed using data samples corresponding to an integrated luminosity of about $614 \mathrm{pb}^{-1}$ collected with the OPAL detector at LEP at centre-of-mass energies between $189 \mathrm{GeV}$ and $209 \mathrm{GeV}$. No evidence for a signal has been observed. A mass limit of $98.5 \mathrm{GeV} / c^{2}$ at the $95 \%$ confidence level has been set for the doubly charged Higgs particle in left-right symmetric models. This is the first search for doubly charged Higgs bosons at centre-of-mass energies larger than $91 \mathrm{GeV}$.
\end{abstract}

To be submitted to Phys. Lett. B 


\section{The OPAL Collaboration}

G. Abbiendi ${ }^{2}$, C. Ainsley ${ }^{5}$, P.F. Åkesson ${ }^{3}$, G. Alexander ${ }^{22}$, J. Allison ${ }^{16}$, G. Anagnostou ${ }^{1}$, K.J. Anderson ${ }^{9}$, S. Arcelli ${ }^{17}$, S. Asai ${ }^{23}$, D. Axen ${ }^{27}$, G. Azuelos ${ }^{18, a}$, I. Bailey ${ }^{26}$, E. Barberio ${ }^{8}$, R.J. Barlow ${ }^{16}$, R.J. Batley ${ }^{5}$, P. Bechtle ${ }^{25}$, T. Behnke ${ }^{25}$, K.W.Bell ${ }^{20}$, P.J. Bell ${ }^{1}$, G. Bella ${ }^{22}$, A. Bellerive ${ }^{6}$, G. Benelli ${ }^{4}$, S. Bethke ${ }^{32}$, O. Biebel ${ }^{32}$, I.J. Bloodworth ${ }^{1}$, O. Boeriu ${ }^{10}$, P. Bock ${ }^{11}$, J. Böhme ${ }^{25}$, D. Bonacorsi ${ }^{2}$, M. Boutemeur ${ }^{31}$, S. Braibant ${ }^{8}$, L. Brigliadori ${ }^{2}$, R.M. Brown ${ }^{20}$, H.J. Burckhart ${ }^{8}$, J. Cammin ${ }^{3}$, S. Campana ${ }^{4}$, R.K. Carnegie ${ }^{6}$, B. Caron ${ }^{28}$, A.A. Carter ${ }^{13}$, J.R. Carter ${ }^{5}$, C.Y. Chang ${ }^{17}$, D.G. Charlton ${ }^{1, b}$, P.E.L. Clarke ${ }^{15}$, E. Clay ${ }^{15}$, I. Cohen ${ }^{22}$, J. Couchman ${ }^{15}$, A. Csilling ${ }^{8, i}$, M. Cuffiani ${ }^{2}$, S. Dado ${ }^{21}$, G.M. Dallavalle ${ }^{2}$, S. Dallison ${ }^{16}$, A. De Roeck $^{8}$, E.A. De Wolf ${ }^{8}$, P. Dervan ${ }^{15}$, K. Desch ${ }^{25}$, B. Dienes ${ }^{30}$, M. Donkers ${ }^{6}$, J. Dubbert ${ }^{31}$, E. Duchovni ${ }^{24}$, G. Duckeck ${ }^{31}$, I.P. Duerdoth ${ }^{16}$, E. Etzion ${ }^{22}$, F. Fabbri ${ }^{2}$, L. Feld ${ }^{10}$, P. Ferrari ${ }^{12}$, F. Fiedler ${ }^{8}$, I. Fleck ${ }^{10}$, M. Ford ${ }^{5}$, A. Frey ${ }^{8}$, A. Fürtjes ${ }^{8}$, D.I. Futyan ${ }^{16}$, P. Gagnon ${ }^{12}$, J.W. Gary ${ }^{4}$, G. Gaycken ${ }^{25}$, C. Geich-Gimbel ${ }^{3}$, G. Giacomelli ${ }^{2}$, P. Giacomelli ${ }^{2}$, M. Giunta ${ }^{4}$, J. Goldberg ${ }^{21}$,

K. Graham ${ }^{26}$, E. Gross ${ }^{24}$, J. Grunhaus ${ }^{22}$, M. Gruwé ${ }^{8}$, P.O. Günther ${ }^{3}$, A. Gupta ${ }^{9}$, C. Hajdu ${ }^{29}$,

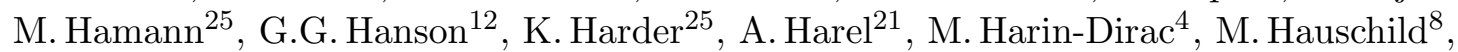
J.Hauschildt ${ }^{25}$, C.M. Hawkes ${ }^{1}$, R. Hawkings ${ }^{8}$, R.J.Hemingway ${ }^{6}$, C. Hensel ${ }^{25}$, G. Herten ${ }^{10}$, R.D.Heuer ${ }^{25}$, J.C. Hill ${ }^{5}$, K. Hoffman ${ }^{9}$, R.J.Homer ${ }^{1}$, D. Horváth ${ }^{29, c}$, K.R. Hossain ${ }^{28}$, R. Howard ${ }^{27}$,

P. Hüntemeyer ${ }^{25}$, P. Igo-Kemenes ${ }^{11}$, K. Ishii ${ }^{23}$, A. Jawahery ${ }^{17}$, H. Jeremie ${ }^{18}$, C.R. Jones ${ }^{5}$, P. Jovanovic ${ }^{1}$, T.R. Junk ${ }^{6}$, N. Kanaya ${ }^{26}$, J. Kanzaki ${ }^{23}$, G. Karapetian ${ }^{18}$, D. Karlen ${ }^{6}$, V. Kartvelishvili ${ }^{16}$, K. Kawagoe ${ }^{23}$, T. Kawamoto ${ }^{23}$, R.K. Keeler ${ }^{26}$, R.G.Kellogg ${ }^{17}$, B.W. Kennedy ${ }^{20}$, D.H. Kim ${ }^{19}$, K.Klein ${ }^{11}$, A.Klier ${ }^{24}$, S. Kluth ${ }^{32}$, T. Kobayashi ${ }^{23}$, M. Kobel ${ }^{3}$, T.P. Kokott ${ }^{3}$, S. Komamiya ${ }^{23}$, R.V.Kowalewski ${ }^{26}$, T. Krämer ${ }^{25}$, T. Kress ${ }^{4}$, P. Krieger ${ }^{6,0}$, J. von Krogh ${ }^{11}$, D. Krop ${ }^{12}$, T. Kuhl ${ }^{25}$, M. Kupper ${ }^{24}$, P. Kyberd ${ }^{13}$, G.D. Lafferty ${ }^{16}$, H. Landsman ${ }^{21}$, D. Lanske ${ }^{14}$, I. Lawson ${ }^{26}$, J.G. Layter ${ }^{4}$, A. Leins ${ }^{31}$, D. Lellouch ${ }^{24}$, J. Letts ${ }^{12}$, L. Levinson ${ }^{24}$, J. Lillich ${ }^{10}$, C. Littlewood ${ }^{5}$, S.L. Lloyd ${ }^{13}$, F.K. Loebinger ${ }^{16}$, J. Lu ${ }^{27}$, J. Ludwig ${ }^{10}$, A. Macchiolo ${ }^{18}$,

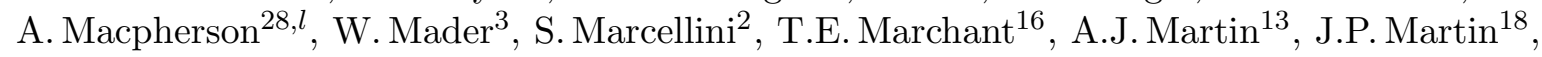
G. Martinez ${ }^{17}$, G. Masetti ${ }^{2}$, T. Mashimo ${ }^{23}$, P. Mättig ${ }^{24}$, W.J. McDonald ${ }^{28}$, J. McKenna ${ }^{27}$,

T.J.McMahon ${ }^{1}$, R.A. McPherson ${ }^{26}$, F. Meijers ${ }^{8}$, P. Mendez-Lorenzo ${ }^{31}$, W. Menges ${ }^{25}$, F.S.Merritt ${ }^{9}$, H. Mes ${ }^{6, a}$, A. Michelini ${ }^{2}$, S. Mihara ${ }^{23}$, G. Mikenberg ${ }^{24}$, D.J.Miller ${ }^{15}$, S. Moed ${ }^{21}$, W. Mohr ${ }^{10}$,

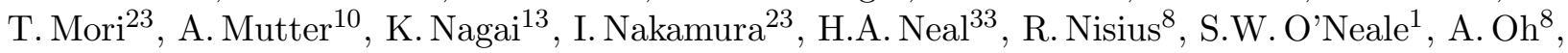
A. Okpara ${ }^{11}$, M.J. Oreglia ${ }^{9}$, S. Orito ${ }^{23}$, C. Pahl ${ }^{32}$, G. Pásztor ${ }^{8, i}$, J.R. Pater ${ }^{16}$, G.N. Patrick ${ }^{20}$, J.E. Pilcher ${ }^{9}$, J. Pinfold ${ }^{28}$, D.E. Plane ${ }^{8}$, B. Poli ${ }^{2}$, J. Polok ${ }^{8}$, O. Pooth ${ }^{8}$, A. Quadt ${ }^{3}$, K. Rabbertz ${ }^{8}$, C. Rembser ${ }^{8}$, P. Renkel ${ }^{24}$, H. Rick ${ }^{4}$, N. Rodning ${ }^{28}$, J.M. Roney ${ }^{26}$, S. Rosati ${ }^{3}$, K. Roscoe $^{16}$, Y.Rozen ${ }^{21}$, K. Runge ${ }^{10}$, D.R. Rust ${ }^{12}$, K. Sachs ${ }^{6}$, T. Saeki ${ }^{23}$, O. Sahr ${ }^{31}$, E.K.G. Sarkisyan ${ }^{8, m}$, A.D. Schaile ${ }^{31}$, O. Schaile ${ }^{31}$, P. Scharff-Hansen ${ }^{8}$, M. Schröder ${ }^{8}$, M. Schumacher ${ }^{25}$, C. Schwick ${ }^{8}$, W.G. Scott ${ }^{20}$, R.Seuster ${ }^{14, g}$, T.G.Shears ${ }^{8, j}$, B.C.Shen ${ }^{4}$, C.H.Shepherd-Themistocleous ${ }^{5}$, P.Sherwood ${ }^{15}$, A.Skuja ${ }^{17}$, A.M. Smith ${ }^{8}$, G.A.Snow ${ }^{17}$, R. Sobie ${ }^{26}$, S. Söldner-Rembold ${ }^{10, e}$, S. Spagnolo ${ }^{20}$, F. Spano ${ }^{9}$, M. Sproston ${ }^{20}$, A.Stahl ${ }^{3}$, K.Stephens ${ }^{16}$, D. Strom ${ }^{19}$, R. Ströhmer ${ }^{31}$, L. Stumpf ${ }^{26}$, B. Surrow ${ }^{25}$, S. Tarem ${ }^{21}$, M. Tasevsky ${ }^{8}$, R.J. Taylor ${ }^{15}$, R. Teuscher ${ }^{9}$, J. Thomas ${ }^{15}$, M.A. Thomson ${ }^{5}$, E. Torrence ${ }^{19}$, D. Toya ${ }^{23}$, T. Trefzger $^{31}$, A. Tricoli ${ }^{2}$, I. Trigger $^{8}$, Z. Trócsányi $^{30, f}$, E. Tsur ${ }^{22}$, M.F. Turner-Watson ${ }^{1}$, I. Ueda ${ }^{23}$, B. Ujvári ${ }^{30, f}$, B. Vachon ${ }^{26}$, C.F. Vollmer ${ }^{31}$,

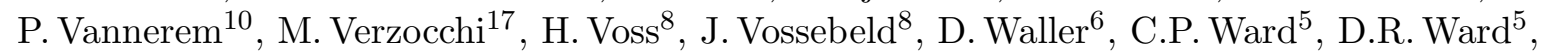
P.M. Watkins ${ }^{1}$, A.T. Watson ${ }^{1}$, N.K. Watson ${ }^{1}$, P.S. Wells ${ }^{8}$, T. Wengler ${ }^{8}$, N. Wermes ${ }^{3}$, D. Wetterling ${ }^{11}$ G.W.Wilson ${ }^{16, n}$, J.A. Wilson ${ }^{1}$, T.R. Wyatt $^{16}$, S. Yamashita ${ }^{23}$, V. Zacek ${ }^{18}$, D. Zer-Zion ${ }^{8, k}$ 
${ }^{1}$ School of Physics and Astronomy, University of Birmingham, Birmingham B15 2TT, UK

2Dipartimento di Fisica dell' Università di Bologna and INFN, I-40126 Bologna, Italy

${ }^{3}$ Physikalisches Institut, Universität Bonn, D-53115 Bonn, Germany

${ }^{4}$ Department of Physics, University of California, Riverside CA 92521, USA

${ }^{5}$ Cavendish Laboratory, Cambridge CB3 0HE, UK

${ }^{6}$ Ottawa-Carleton Institute for Physics, Department of Physics, Carleton University, Ottawa, Ontario K1S 5B6, Canada

${ }^{8}$ CERN, European Organisation for Nuclear Research, CH-1211 Geneva 23, Switzerland

${ }^{9}$ Enrico Fermi Institute and Department of Physics, University of Chicago, Chicago IL 60637, USA

${ }^{10}$ Fakultät für Physik, Albert Ludwigs Universität, D-79104 Freiburg, Germany

${ }^{11}$ Physikalisches Institut, Universität Heidelberg, D-69120 Heidelberg, Germany

${ }^{12}$ Indiana University, Department of Physics, Swain Hall West 117, Bloomington IN 47405, USA

${ }^{13}$ Queen Mary and Westfield College, University of London, London E1 4NS, UK

${ }^{14}$ Technische Hochschule Aachen, III Physikalisches Institut, Sommerfeldstrasse 26-28, D-52056

Aachen, Germany

${ }^{15}$ University College London, London WC1E 6BT, UK

${ }^{16}$ Department of Physics, Schuster Laboratory, The University, Manchester M13 9PL, UK

${ }^{17}$ Department of Physics, University of Maryland, College Park, MD 20742, USA

${ }^{18}$ Laboratoire de Physique Nucléaire, Université de Montréal, Montréal, Quebec H3C 3J7, Canada

${ }^{19}$ University of Oregon, Department of Physics, Eugene OR 97403, USA

${ }^{20}$ CLRC Rutherford Appleton Laboratory, Chilton, Didcot, Oxfordshire OX11 0QX, UK

${ }^{21}$ Department of Physics, Technion-Israel Institute of Technology, Haifa 32000, Israel

${ }^{22}$ Department of Physics and Astronomy, Tel Aviv University, Tel Aviv 69978, Israel

${ }^{23}$ International Centre for Elementary Particle Physics and Department of Physics, University of Tokyo, Tokyo 113-0033, and Kobe University, Kobe 657-8501, Japan

${ }^{24}$ Particle Physics Department, Weizmann Institute of Science, Rehovot 76100, Israel

${ }^{25}$ Universität Hamburg/DESY, II Institut für Experimental Physik, Notkestrasse 85, D-22607 Hamburg, Germany

${ }^{26}$ University of Victoria, Department of Physics, P O Box 3055, Victoria BC V8W 3P6, Canada

${ }^{27}$ University of British Columbia, Department of Physics, Vancouver BC V6T 1Z1, Canada

${ }^{28}$ University of Alberta, Department of Physics, Edmonton AB T6G 2J1, Canada

${ }^{29}$ Research Institute for Particle and Nuclear Physics, H-1525 Budapest, P O Box 49, Hungary

${ }^{30}$ Institute of Nuclear Research, H-4001 Debrecen, P O Box 51, Hungary

${ }^{31}$ Ludwigs-Maximilians-Universität München, Sektion Physik, Am Coulombwall 1, D-85748 Garching, Germany

${ }^{32}$ Max-Planck-Institute für Physik, Föhring Ring 6, 80805 München, Germany

${ }^{33}$ Yale University,Department of Physics,New Haven, CT 06520, USA

${ }^{a}$ and at TRIUMF, Vancouver, Canada V6T 2A3

${ }^{b}$ and Royal Society University Research Fellow

${ }^{c}$ and Institute of Nuclear Research, Debrecen, Hungary

${ }^{e}$ and Heisenberg Fellow

$f$ and Department of Experimental Physics, Lajos Kossuth University, Debrecen, Hungary

$g$ and MPI München

${ }^{i}$ and Research Institute for Particle and Nuclear Physics, Budapest, Hungary

${ }^{j}$ now at University of Liverpool, Dept of Physics, Liverpool L69 3BX, UK 
${ }^{k}$ and University of California, Riverside, High Energy Physics Group, CA 92521, USA

${ }^{l}$ and CERN, EP Div, 1211 Geneva 23

$m$ and Universitaire Instelling Antwerpen, Physics Department, B-2610 Antwerpen, Belgium

${ }^{n}$ now at University of Kansas, Dept of Physics and Astronomy, Lawrence, KS 66045, USA

${ }^{0}$ now at University of Toronto, Dept of Physics, Toronto, Canada 


\section{Introduction}

Doubly charged Higgs bosons $\left(\mathrm{H}^{ \pm \pm}\right)$appear in theories beyond the Standard Model [1]. Many authors have pointed out that these doubly charged scalar particles occur naturally in the leftright symmetric models which allow small neutrino masses [2], and stress the importance of looking for their existence [3]. Supersymmetric left-right models in which the $S U(2)_{\mathrm{R}}$ gauge symmetry is broken by triplet Higgs fields do not conserve baryon and lepton numbers. They also yield a natural embedding of a large mass for the right-handed Majorana neutrino needed for the implementation of the see-saw mechanism for small neutrino masses. Recently, it has pointed out that such models can lead to "light" doubly charged Higgs particles, in the $100 \mathrm{GeV} / \mathrm{c}^{2}$ mass range 沺. An important feature of the doubly charged Higgs bosons is that they couple, at tree level, only to charged leptons and to other Higgs and gauge bosons, in order to conserve electric charge. Thus doubly charged Higgs bosons could be copiously produced in pairs via $\mathrm{e}^{+} \mathrm{e}^{-} \rightarrow \mathrm{H}^{++} \mathrm{H}^{--}$at LEP II with a differential cross section at tree level depending on the doubly charged Higgs boson mass and the centre-of-mass energy of the colliding beams. In left-right symmetric models, two Higgs triplets arise, conventionnaly labelled left and right handed. The cross section of $\mathrm{e}^{+} \mathrm{e}^{-} \rightarrow \mathrm{H}_{\mathrm{L}}^{++} \mathrm{H}_{\mathrm{L}}^{--}$is not equal to that for $\mathrm{e}^{+} \mathrm{e}^{-} \rightarrow \mathrm{H}_{\mathrm{R}}^{++} \mathrm{H}_{\mathrm{R}}^{--}$. The events are characterized by the $\sin ^{2} \theta$ dependence, where $\theta$ is the angle of the $\mathrm{H}^{++}$with respect to the electron beam axis, anticipated for the production of a pair of spin 0 particles via the s-channel $\mathrm{Z}$ or $\gamma$ exchange.

The partial widths of the dominant decay modes, $\mathrm{H}^{++} \rightarrow \ell^{+} \ell^{+}$and $\mathrm{H}^{--} \rightarrow \ell^{-} \ell^{-}$, have been calculated by Huitu and collaborators [5] and by Swartz [6]. For instance for $\mathrm{H}$ decaying into $\tau \tau$ it is, at tree level:

$$
\Gamma_{\tau \tau}\left(\mathrm{H}^{ \pm \pm} \rightarrow \tau^{ \pm} \tau^{ \pm}\right)=\frac{h_{\tau \tau}^{2}}{8 \pi} M_{\mathrm{H}}\left(1-\frac{2 m_{\tau}^{2}}{M_{\mathrm{H}}^{2}}\right)\left(1-\frac{4 m_{\tau}^{2}}{M_{\mathrm{H}}^{2}}\right)^{1 / 2}
$$

where $M_{\mathrm{H}}$ is the mass of the doubly charged Higgs boson, $m_{\tau}$ is the mass of the tau lepton and $h_{\tau \tau}$ is the unknown $\mathrm{H} \tau \tau$ Yukawa coupling constant. If $M_{\mathrm{H}} \gg m_{\tau}$, values of $h_{\tau \tau} \gtrsim 10^{-7}$ can ensure the fast decay of the Higgs particle $\left(\lesssim 10^{-11} \mathrm{~s}\right)$. Observation of a four lepton final state would provide a clean signature for the identification of the event. On the other hand for values of $h_{\tau \tau}$ smaller than about $10^{-7}$ long lived doubly charged Higgs bosons could be detected as events with large impact parameter tracks, kinked tracks, or stable doubly charged massive particles. OPAL has searched for charged massive particles at centre-of-mass energies up to $183 \mathrm{GeV}$ [7].

Previous searches for $\mathrm{H}^{ \pm \pm}$pair production have been performed with the MARKII [8] and the OPAL [9] detectors using data collected near the $\mathrm{Z}$ peak and have excluded the existence of the $\mathrm{H}^{ \pm \pm}$with mass less than $45.6 \mathrm{GeV} / \mathrm{c}^{2}$ and for values of $h_{\tau \tau}$ that extend down to zero, apart from a small band around $h_{\tau \tau} \sim 10^{-7}$.

Significant couplings to electrons or muons are not likely as there are indirect constraints [6, 10] on the decays of $\mathrm{H}^{++} \rightarrow \mathrm{e}^{+} \mathrm{e}^{+}$from high energy Bhabha scattering, of $\mathrm{H}^{++} \rightarrow \mu^{+} \mu^{+}$from the absence of muonium - anti-muonium transitions, and of $\mathrm{H}^{++} \rightarrow \mathrm{e}^{+} \mu^{+}$from limits on the flavour changing decay $\mu^{-} \rightarrow \mathrm{e}^{+} \mathrm{e}^{-} \mathrm{e}^{-}$. In this paper, however, we will present our results based on general searches for $\mathrm{e}^{+} \mathrm{e}^{-} \rightarrow$ four leptons with missing energy (A), and four leptons without missing energy (B). We have considered the following channels: (A) $\mathrm{H}^{++} \mathrm{H}^{--} \rightarrow \tau^{+} \tau^{+} \tau^{-} \tau^{-}, \mathrm{e}^{+} \mathrm{e}^{+} \tau^{-} \tau^{-}$and $\mu^{+} \mu^{+} \tau^{-} \tau^{-}$, and (B) $\mathrm{H}^{++} \mathrm{H}^{--} \rightarrow \mathrm{e}^{+} \mathrm{e}^{+} \mathrm{e}^{-} \mathrm{e}^{-}, \mu^{+} \mu^{+} \mu^{-} \mu^{-}$and $\mathrm{e}^{+} \mathrm{e}^{+} \mu^{-} \mu^{-}$.

The data set is from $\mathrm{e}^{+} \mathrm{e}^{-}$collisions recorded with the OPAL detector at LEP, and is composed of events at centre-of-mass energies between $\sqrt{s}=189 \mathrm{GeV}$ and $209 \mathrm{GeV}$ with a total integrated 
luminosity of about $614 \mathrm{pb}^{-1}$. A complete description of the OPAL detector can be found in 11 , 12, 13].

\section{Monte Carlo Simulation}

Monte Carlo samples are used to model the pair production of doubly charged Higgs bosons as well as to estimate the expected background due to Standard Model processes.

The simulation of the signal events with one non-zero $h_{\ell \ell^{\prime}}$ coupling at a time and with zero lifetime has been done with the Monte Carlo generator PYTHIA [14] modified according to [5]. $\mathrm{H}^{++} \mathrm{H}^{--}$ events have been generated for various mass points ranging from $45 \mathrm{GeV} / c^{2}$ to half the centre-ofmass energy, $\sqrt{s}$. At each point on the $\left(M_{\mathrm{H}}, \sqrt{s}\right)$ plane, 10000 events for each of the six $\mathrm{H}^{ \pm \pm} \rightarrow \ell^{ \pm} \ell^{ \pm}$ decays have been generated.

The main sources of background arise from Standard Model four-fermion final states $\left(\mathrm{e}^{+} \mathrm{e}^{-} \rightarrow 4 \mathrm{f}\right)$ and a small contribution from two-photon $\left(\mathrm{e}^{+} \mathrm{e}^{-} \rightarrow \gamma \gamma \rightarrow\right.$ hadrons, $\left.\ell^{+} \ell^{-}\right)$and two-fermion $\left(\mathrm{e}^{+} \mathrm{e}^{-} \rightarrow\right.$ $\mathrm{Z} \rightarrow \mathrm{ff}$ ) processes. For two-photon processes, the PHOJET [15], PYTHIA [14], and HERWIG [16] generators have been used to simulate hadronic final states. The Vermaseren [17] generator has been used to estimate the background contribution from all two-photon $\mathrm{e}^{+} \mathrm{e}^{-} \ell^{+} \ell^{-}$final states. All other four-fermion final states have been simulated with grc4f [18]. For the two-fermion final states, BHWIDE [19] was used for the ee( $\gamma$ ) final state, and KORALZ [20] and KK2f [21] for the $\mu \mu$ and $\tau \tau$ states. The multi-hadronic events, $q q(\gamma)$, were simulated using PYTHIA and KK2f.

All events were processed through the full simulation of the OPAL detector [22], with the same analysis chain being applied to simulated events and to data.

\section{Data Analysis}

The final states resulting from $\mathrm{e}^{+} \mathrm{e}^{-} \rightarrow \mathrm{H}^{++} \mathrm{H}^{--}$processes followed by $\mathrm{H}^{ \pm \pm} \rightarrow \ell^{ \pm} \ell^{ \pm}$decays consist of four leptons. Two different analyses are applied depending on the decay assumed: for $\mathrm{H}^{ \pm \pm} \rightarrow \tau^{ \pm} \mathrm{e}^{ \pm}$, $\tau^{ \pm} \mu^{ \pm}$or $\tau^{ \pm} \tau^{ \pm}$, a selection for four lepton candidates plus missing energy (resulting from the decays of the tau leptons) is applied, while for $\mathrm{H}^{ \pm \pm} \rightarrow \mathrm{e}^{ \pm} \mathrm{e}^{ \pm}, \mu^{ \pm} \mu^{ \pm}$or $\mathrm{e}^{ \pm} \mu^{ \pm}$, a selection for four leptons (electrons or muons) without missing energy is used. Although lepton identification is used in the event selection, it is not used for splitting the selected events into the different channels within the two analyses.

The analyses are similar to the R-parity violation search [23, 24] that considers four leptons with or without missing energy topologies. These event selection procedures are described in detail in [23, 24] and are only briefly outlined below. The cut values used in the present analysis are optimized for the searches for $\mathrm{H}^{ \pm \pm} \rightarrow \tau^{ \pm} \tau^{ \pm}$(in the missing energy analysis) and for $\mathrm{H}^{ \pm \pm} \rightarrow \mathrm{e}^{ \pm} \mathrm{e}^{ \pm}$(in the no missing energy analysis). The selections are then applied as such for the other channels considered, and the efficiencies are calculated separately for each channel.

The visible energy and the momentum of the event are calculated using the method described in 25$]$. A preselection is applied, consisting of

- Data quality requirements as described in [26]; 
- Vetoes on the energy deposited in the forward detectors to reduce background due to twophoton events and interactions of beam particles with the beam pipe or residual gas: the energy deposited in each silicon-tungsten forward calorimeter and in each forward detector has to be less than $5 \mathrm{GeV}$ (these detectors are located in the forward region, with polar angle $|\cos \theta|>0.99$, surrounding the beam pipe); there should be no signal in the MIP plug scintillators 2 .

- A requirement that at least four and no more than 10 tracks are reconstructed.

After the preselection, a series of cuts is applied as follows.

For the missing energy selection:

(A1) In order to reduce the background from two-photon processes and from radiative return events $\left(\mathrm{e}^{+} \mathrm{e}^{-} \rightarrow \mathrm{Z} \gamma\right)$ with the $\gamma$ escaping into the beam pipe:

- The event's transverse momentum, $p_{\mathrm{t}}$, is required to be larger than $0.035 \sqrt{s} / c$.

- The polar angle, $\theta_{\text {miss }}$, of the missing momentum direction is required to satisfy $\left|\cos \theta_{\text {miss }}\right|<0.9$.

- The event's longitudinal momentum, $p_{\mathrm{z}}^{\text {miss }}$, is required to be smaller than $0.25 \sqrt{s} / c$.

(A2) The visible energy, $E_{\text {vis }}$, of the event is required to be between $0.35 \sqrt{s}$ and $0.85 \sqrt{s}$.

(A3) There have to be at least three tracks with a transverse momentum with respect to the beam axis larger than $1.5 \mathrm{GeV} / c$.

(A4) There have to be at least three well identified isolated leptons [24] (e, $\mu, \tau)$, each with a transverse momentum larger than $1.5 \mathrm{GeV} / c$.

(A5) The total leptonic momentum, $p_{\text {lept }}$, defined as the scalar sum of the momenta of all identified leptons, is required to be greater than $0.40 E_{\text {vis }} / c$.

For the no missing energy selection:

(B1) The polar angle, $\theta_{\text {miss }}$, of the missing momentum direction has to satisfy $\left|\cos \theta_{\text {miss }}\right|<0.9$, in order to reject events in which a possible missing momentum points towards the beam pipe.

(B2) $E_{\text {vis }}$ is required to be larger than $0.90 \sqrt{s}$.

(B3) There have to be at least three isolated tracks each with a transverse momentum larger than $1.5 \mathrm{GeV} / c$.

(B4) There have to be at least three well identified isolated leptons [24] (e, $\mu, \tau)$, each with a transverse momentum larger than $1.5 \mathrm{GeV} / c$.

(B5) The total leptonic momentum is required to be greater than $0.60 E_{\text {vis }} / c$.

\footnotetext{
${ }^{1} \mathrm{~A}$ right-handed coordinate system is adopted, where the $x$-axis points to the centre of the LEP ring, and positive $z$ is along the electron beam direction. The angles $\theta$ and $\phi$ are the polar and azimuthal angles, respectively.

${ }^{2}$ The MIP plug scintillators [13] are an array of thin scintillating tiles with embedded wavelength shifting fibre readout which have been installed to improve the hermiticity of the detector. They cover the polar angular range between 43 and 200 mrad.
} 
(B6) In order to select events with a pair of particles with identical masses, the following procedure is applied. Pairs are formed using the four most energetic tracks, and the invariant mass is computed for each pair. Events are selected if one of the three possible pairings satisfies $\left|m_{i, j}-m_{k, l}\right| /\left(m_{i, j}+m_{k, l}\right)<0.3$, where $m_{i, j}$ is the invariant mass of the pair $(i, j)$. Only pairs with invariant masses $m_{i, j}$ greater than $20 \mathrm{GeV} / c^{2}$ are used in the computation.

The numbers of events remaining after each cut are listed in Tables 1 and 2. The poor agreement between data and Monte Carlo expectation in the early stages of the selections is due to the beamrelated backgrounds and incomplete modelling of two-photon processes. When the background from these processes has been effectively reduced, the agreement between data and Monte Carlo is satisfactory.

Figure 11 shows the distributions of some of the variables used in the selections for the data and the background simulation (the contributions from all centre-of-mass energies are added). For the missing energy selection, figure 1 1a) shows the distributions of the number of tracks with a transverse momentum with respect to the beam axis larger than $1.5 \mathrm{GeV} / c$ after cut (A2) has been applied. The distributions for a signal sample for $M_{\mathrm{H}}=90 \mathrm{GeV} / c^{2}$ and $\mathrm{H}^{ \pm \pm} \rightarrow \tau^{ \pm} \tau^{ \pm}$at $\sqrt{s}=206 \mathrm{GeV}$ are also shown with arbitrary normalisation. Similarly, figure 1 $\mathrm{b}$ ) shows the distributions of the total leptonic momentum divided by the visible energy of the event after all but cut (A5) have been applied.

For the no missing energy selection, figure 11 c) shows the distributions of the number of well identified isolated leptons after cut (B3) and figure 11d) the distributions of the total leptonic momentum divided by the visible energy of the event after cut (B4). The distributions for a signal sample for $M_{\mathrm{H}}=90 \mathrm{GeV} / c^{2}$ and $\mathrm{H}^{ \pm \pm} \rightarrow \mathrm{e}^{ \pm} \mathrm{e}^{ \pm}$at $\sqrt{s}=206 \mathrm{GeV}$ are also shown with arbitrary normalisation.

The signal detection efficiencies are given in Table 3. For values of $h_{\ell \ell}$ smaller than approximately $10^{-7}$, the efficiency to detect $\mathrm{H}^{ \pm \pm} \rightarrow \ell^{ \pm} \ell^{ \pm}$decreases due to the long decay length of the $\mathrm{H}^{ \pm \pm}$. At very small $h_{\ell \ell}\left(10^{-8}\right.$ to $\left.10^{-9}\right)$ the $\mathrm{H}^{ \pm \pm}$could traverse the tracking chambers of the detector without decaying. We assume zero detection efficiency for values of $h_{\ell \ell}$ smaller than $10^{-7}$.

\section{Inefficiencies and Systematic Uncertainties}

The following systematic errors on the signal detection efficiencies have been considered: the statistical uncertainty on the determination of the efficiency from the Monte Carlo simulation (less than 1\% absolute uncertainty); the systematic uncertainty on the measurement of the integrated luminosity $(0.20 \%$ to $0.24 \%)$; and the errors due to the modelling of the physics variables (between $0.5 \%$ and $4.5 \%$ absolute uncertainty). These last errors are determined by shifting each cut by the maximum amount such that distributions from high statistics Monte Carlo samples remain in agreement with the data.

For the expected number of background events, an uncertainty due to Monte Carlo statistics (4\% to $8 \%$ ) and a systematic error due to the modelling of the physics variables ( $5 \%$ to $7 \%$ ) have been taken into account.

The total systematic uncertainty was calculated by summing in quadrature the individual errors. Correlations between systematic uncertainties at different centre-of-mass energies have been taken into account. 
In addition to effects included in the detector simulation, a relative efficiency loss of $1.5 \%$ to $2.9 \%$ arises from beam-related background in the silicon-tungsten forward calorimeter and in the forward detector. This is estimated using random beam crossing events.

\section{Interpretation}

As shown in Table 3 the observed numbers of events selected by the missing energy and the no missing energy selections (17 and 10) are consistent with the numbers expected from Standard Model processes $(25.19 \pm 0.92 \pm 1.06$ and $6.57 \pm 0.50 \pm 0.31)$. No evidence for $\mathrm{H}^{++} \mathrm{H}^{--}$production has therefore been observed and limits on the doubly charged Higgs boson production cross-section and mass have been derived.

For each selected event, a reconstructed mass is computed. Figure 2 shows data and background as well as typical signal reconstructed mass distributions for the $\mathrm{e}^{+} \mu^{+} \mathrm{e}^{-} \mu^{-}$and $\tau^{+} \tau^{+} \tau^{-} \tau^{-}$states. The momenta of the four lepton candidates are constrained so that the total energy is the centre-of-mass energy, and the total momentum adds to zero. For events with unambiguous charge assignments (two positive lepton candidates and two negative lepton candidates), the charge is used to assign leptons to the hypothesized doubly-charged Higgs bosons. The reconstructed mass is then the average of the invariant masses reconstructed for the two boson hypotheses in the event. For events for which the charge assignment is ambiguous (three or more lepton candidates of the same sign), the assignment of lepton candidates to hypothesized Higgs bosons is chosen to minimize the difference between the two reconstructed masses of the Higgs bosons, and the average reconstructed mass of the two bosons is used as the event reconstructed mass. In the channel without missing energy, the reconstructed mass resolution is characterized by two components: a core of width $400 \mathrm{MeV} / \mathrm{c}^{2}$ with approximately $96 \%$ of the signal, and a tail of width $3 \mathrm{GeV} / c^{2}$, with approximately $4 \%$ of the signal. In the channel with missing energy, for the $\tau^{+} \tau^{+} \tau^{-} \tau^{-}$final state, for example, approximately $40 \%$ of the signal is concentrated within a peak of width $2.5 \mathrm{GeV} / c^{2}$, while the remainder has a long tail. The first bin of the histograms collects events which fail to have a reconstructed mass (e.g. missing a lepton candidate), and the last bin contains all overflows (events with a reconstructed mass larger than $130 \mathrm{GeV} / c^{2}$.

The distributions of the reconstructed masses of signal Monte Carlo events for various test masses are formed, with bin widths of $500 \mathrm{MeV} / c^{2}$. To obtain the expected signal distribution for an arbitrary test mass, the available signal histograms are interpolated [27]. Histograms of the reconstructed masses of Standard Model Monte Carlo events, averaging over $22 \mathrm{GeV} / c^{2}$ wide mass intervals, are used as the background.

A likelihood ratio method 28] has been used to determine an upper limit for the production crosssection. This method combines the results obtained at different centre-of-mass energies, taking into account the number of candidates and their reconstructed masses, the signal detection efficiencies, the expected number of background events and the distributions of the reconstructed masses for background and for signal events. The confidence levels are computed by binning the data in the reconstructed mass separately for the different centre-of-mass energies 5 , and combining them. Systematic uncertainties on the efficiencies and on the number of expected background events have been taken into account in the limit calculation according to [29].

Within supersymmetric left-right symmetric models, this results in limits on $\sigma\left(\mathrm{e}^{+} \mathrm{e}^{-} \rightarrow \mathrm{H}^{++} \mathrm{H}^{--}\right) \times$

\footnotetext{
${ }^{3}$ When calculating limits, cross-sections at different $\sqrt{s}$ are estimated by weighting by $\beta^{3} / s$, where $\beta$ is $p_{\mathrm{H}^{ \pm \pm}} / \mathrm{E}_{\mathrm{beam}}$
} 
$B R^{2}\left(\mathrm{H}^{ \pm \pm} \rightarrow \ell^{ \pm} \ell^{\prime \pm}\right)$ given as a function of the $\mathrm{H}^{ \pm \pm}$mass, where $B R\left(\mathrm{H}^{ \pm \pm} \rightarrow \ell^{ \pm} \ell^{\prime}\right)$ is the branching ratio of the decay $\mathrm{H}^{ \pm \pm} \rightarrow \ell^{ \pm} \ell^{\prime \pm}$. Figure 3 shows the $95 \%$ confidence level upper limit on $\sigma\left(\mathrm{e}^{+} \mathrm{e}^{-} \rightarrow\right.$ $\left.\mathrm{H}^{++} \mathrm{H}^{--}\right) \times B R^{2}\left(\mathrm{H}^{ \pm \pm} \rightarrow \ell^{ \pm} \ell^{\prime \pm}\right)$ for each of the six possible leptonic decays assuming conservatively that the efficiency for all other decays is negligible.

As an example, Figure 4 shows the 95\% confidence level upper limit on the cross-section for pair production of $\mathrm{H}^{ \pm \pm}$followed by a decay into $\tau^{ \pm} \tau^{ \pm}$at $\sqrt{s}=206 \mathrm{GeV}$, obtained from the data collected at centre-of-mass energies between $189 \mathrm{GeV}$ and $209 \mathrm{GeV}$. Comparing this limit with the expected cross-sections for left-handed $\mathrm{H}_{\mathrm{L}}^{ \pm \pm}$and right-handed $\mathrm{H}_{\mathrm{R}}^{ \pm \pm}$pair production [5], $\sigma_{\mathrm{L}}$ and $\sigma_{\mathrm{R}}$, yields $95 \%$ C.L. lower mass limits of $99.0 \mathrm{GeV} / c^{2}$ and $98.5 \mathrm{GeV} / c^{2}$, respectively, assuming a $100 \%$ decay into $\tau^{ \pm} \tau^{ \pm}$. As listed in Table 4 the left-handed $\mathrm{H}_{\mathrm{L}}^{ \pm \pm}$mass limits for all channels taken independently lie between $99.0 \mathrm{GeV} / c^{2}$ (for $100 \%$ decay into $\tau^{ \pm} \tau^{ \pm}$) and $100.5 \mathrm{GeV} / c^{2}$ (for $\mu^{ \pm} \mu^{ \pm}$). For right-handed $\mathrm{H}_{\mathrm{R}}^{ \pm \pm}$the mass limits lie between $98.5 \mathrm{GeV} / c^{2}$ and $100.1 \mathrm{GeV} / c^{2}$.

\section{Conclusion}

The OPAL data sets at centre-of-mass energies between $189 \mathrm{GeV}$ and $209 \mathrm{GeV}$, corresponding to a total luminosity of about $614 \mathrm{pb}^{-1}$, have been searched for evidence of the reaction $\mathrm{e}^{+} \mathrm{e}^{-} \rightarrow \mathrm{H}^{++} \mathrm{H}^{--}$ followed by the decays $\mathrm{H}^{ \pm \pm} \rightarrow \ell^{ \pm} \ell^{\prime \pm}$ in R-parity conserving supersymmetric left-right symmetric models. No significant excess of events has been observed in the data. Production cross-section limits have been derived for values of the Yukawa coupling constant $h_{\ell \ell^{\prime}}$ larger than $10^{-7}$. A lower mass limit of $98.5 \mathrm{GeV} / c^{2}$ at the $95 \%$ confidence level has been obtained for Higgs bosons decaying via a single channel $\mathrm{H}^{ \pm \pm} \rightarrow \ell^{ \pm} \ell^{\prime \pm}$ with $100 \%$ branching ratio.

\section{Acknowledgements}

We particularly wish to thank the SL Division for the efficient operation of the LEP accelerator at all energies and for their close cooperation with our experimental group. We thank our colleagues from CEA, DAPNIA/SPP, CE-Saclay for their efforts over the years on the time-of-flight and trigger systems which we continue to use. We also gratefully thank R.N. Mohapatra for valuable support on theoretical issues.

In addition to the support staff at our own institutions we are pleased to acknowledge the

Department of Energy, USA,

National Science Foundation, USA,

Particle Physics and Astronomy Research Council, UK,

Natural Sciences and Engineering Research Council, Canada,

Israel Science Foundation, administered by the Israel Academy of Science and Humanities,

Minerva Gesellschaft,

Benoziyo Center for High Energy Physics,

Japanese Ministry of Education, Science and Culture (the Monbusho) and a grant under the Monbusho International Science Research Program,

Japanese Society for the Promotion of Science (JSPS),

German Israeli Bi-national Science Foundation (GIF),

Bundesministerium für Bildung und Forschung, Germany,

National Research Council of Canada, 
Research Corporation, USA,

Hungarian Foundation for Scientific Research, OTKA T-029328, T023793 and OTKA F-023259, Fund for Scientific Research, Flanders, F.W.O.-Vlaanderen, Belgium. 


\section{References}

[1] G.B. Gelmini and M. Roncadedli, Phys. Lett. B99 (1981) 411;

R.N. Mohapatra and J.D. Vergados, Phys. Rev. Lett. 47 (1981) 1713;

V. Barger, H. Baer, W.Y. Keung and R.J.N. Phillips, Phys. Rev. D26 (1982) 218.

[2] R.N. Mohapatra and G. Senjanovic, Phys. Rev. D23 (1981) 165.

[3] T.G. Rizzo, Phys. Rev. D25 (1982) 1355;

M. Lusignoli and S. Petrarca, Phys. Lett. B226 (1989) 397;

J.F. Gunion, Int. J. Mod. Phys. A11 (1996) 1551.

[4] C. S. Aulakh, A. Melfo and G. Senjanovic, Phys. Rev. D57 (1998) 4174;

Z. Chacko and R. N. Mohapatra, Phys. Rev. D58 (1998) 15003;

B. Dutta and R. N. Mohapatra, Phys. Rev. D59 (1999) 15018.

[5] K. Huitu et al., "Decays and Production of Doubly Charged Higgses: Collection of Formulas for Simulation Studies", Int. Report Helsinki Institute of Physics 1998-06.

[6] M.L. Swartz, Phys. Rev. D40 (1989) 1521.

[7] OPAL Collab. K. Ackerstaff et al., Phys. Lett. B433 (1998) 195.

[8] M. Swartz et al., Phys. Rev. Lett. 64 (1990) 2877.

[9] OPAL Collab. P. D. Acton et al., Phys. Lett. B295 (1992) 347.

[10] K. Huitu et al., Nucl. Phys. B487 (1997) 27.

[11] OPAL Collab., K. Ahmet et al., Nucl. Instr. Meth. A305 (1991) 275;

S. Anderson et al., Nucl. Instr. Meth. A403 (1998) 326.

[12] B.E. Anderson et al., IEEE Transactions on Nuclear Science 41 (1994) 845.

[13] G. Aguillion et al., Nucl. Instr. Meth. A417 (1998) 277.

[14] T. Sjöstrand and M. Bengtsson, Comp. Phys. Comm. 43 (1987) 367;

"PYTHIA 5.6 and JETSET 7.3, Physics and Manual", CERN-TH. 6488/92;

T. Sjöstrand, Comp. Phys. Comm. 82 (1994) 74.

[15] R. Engel and J. Ranft, Phys. Rev. D54 (1996) 4244;

R. Engel, Z. Phys. C66 (1995) 203.

[16] G. Marchesini et al., Comp. Phys. Comm. 67 (1992) 465.

[17] R. Bhattacharya, J. Smith and G. Grammer, Phys. Rev. D15 (1977) 3267;

J. Smith, J.A.M. Vermaseren and G. Grammer, Phys. Rev. D15 (1977) 3280.

[18] J. Fujimoto et al., Comp. Phys. Comm. 100 (1997) 128.

[19] S. Jadach et al., in "Physics at LEP2", Editors: G. Altarelli, T. Sjöstrand and F. Zwirner, CERN 96-01, vol.2, p. 229;

S. Jadach, W. Placzek and B.F.L. Ward, Phys. Lett. B390 (1997) 298. 
[20] S. Jadach, B.F.L. Ward and Z. Wa̧s, Comp. Phys. Comm. 79 (1994) 503.

[21] S. Jadach, B.F. Ward and Z. Was, Phys. Lett. B449 (1999) 97;

S. Jadach, B.F.L. Ward and Z. Wạs, Comp. Phys. Comm. 130 (2000) 260.

[22] J. Allison et al., Nucl. Instr. Meth. A317 (1992) 47.

[23] OPAL Collab. G. Abbiendi et al., Eur. Phys. J. C11 (1999) 619.

[24] OPAL Collab. G. Abbiendi et al., Eur. Phys. J. C12 (2000) 1.

[25] OPAL Collab., M.Z. Akrawy et al., Phys. Lett. B253 (1991) 511.

[26] OPAL Collab., R. Akers et al., Z. Phys. C61 (1994) 19.

[27] A. L. Read, Nucl. Instr. Meth. A425 (1999) 357.

[28] A. G. Frodesen, O. Skeggestad, and H. Tofte, "Probability and Statistics in Particle Physics", Universitetsforlaget, 1979, ISBN 82-00-01-01906-3;

S. L. Meyer, "Data Analysis for Scientists and Engineers", John Wiley and Sons, 1975, ISBN 0-471-59995-6;

A. L. Read, "Modified Frequentist Analysis of Search Results (The CL(S) Method)", CERNOPEN-2000-205, In 1st workshop on Confidence Limits (2000) 81;

T. R. Junk, Nucl. Instr. Meth. Phys. Res., A434 (1999) 435.

[29] R. D. Cousins and V. L. Highland, Nucl. Instr. Meth. A320 (1992) 331. 


\begin{tabular}{|l||r||r||r|r|r|r||r|}
\hline Cut & data & total bkg & $\ell^{+} \ell^{-}(\gamma)$ & $\mathrm{q} \overline{\mathrm{q}}(\gamma)$ & $' \gamma \gamma^{\prime}$ & $4-\mathrm{f}$ & signal \\
\hline \hline Pres. & 80543 & 51158.7 & 1667.5 & 1210.5 & 47580.5 & 700.2 & $86.8 \%$ \\
A1 & 1550 & 1318.1 & 676.6 & 194.6 & 156.5 & 290.4 & $73.9 \%$ \\
$\mathrm{~A} 2$ & 684 & 601.0 & 379.3 & 28.3 & 6.8 & 186.5 & $62.7 \%$ \\
$\mathrm{~A} 3$ & 417 & 375.0 & 230.3 & 17.7 & 3.0 & 123.9 & $54.0 \%$ \\
$\mathrm{~A} 4$ & 25 & 30.0 & 5.6 & 0.5 & 0.9 & 22.9 & $47.7 \%$ \\
A5 & 17 & 25.2 & 5.3 & 0.1 & 0.9 & 18.9 & $44.5 \%$ \\
& & \pm 1.4 & \pm 0.4 & \pm 0.1 & \pm 0.5 & \pm 1.2 & $\pm 1.0 \%$ \\
\hline
\end{tabular}

Table 1: The remaining numbers of events after each cut of the missing energy selection for various background processes normalised to $614 \mathrm{pb}^{-1}$ are compared with data collected at $\sqrt{s}$ between $189 \mathrm{GeV}$ and $209 \mathrm{GeV}$. Efficiencies for a simulated event sample of $\mathrm{H}^{++} \mathrm{H}^{--}$with $\mathrm{H}^{ \pm \pm} \rightarrow \tau^{ \pm} \tau^{ \pm}$ and $M_{\mathrm{H}}=90 \mathrm{GeV} / c^{2}$ at $\sqrt{s}=206 \mathrm{GeV}$ are also given.

\begin{tabular}{|l||r||r||r|r|r|r||r|}
\hline Cut & data & total bkg & $\ell^{+} \ell^{-}(\gamma)$ & $\mathrm{q} \overline{\mathrm{q}}(\gamma)$ & $' \gamma \gamma$ & $4-\mathrm{f}$ & signal \\
\hline \hline Pres. & 80543 & 51158.7 & 1667.5 & 1210.5 & 47580.5 & 700.2 & $84.7 \%$ \\
B1 & 20655 & 13878.8 & 909.9 & 271.8 & 12341.4 & 355.7 & $71.9 \%$ \\
B2 & 571 & 557.3 & 277.5 & 204.4 & 5.0 & 70.3 & $71.3 \%$ \\
B3 & 53 & 50.9 & 5.2 & 26.2 & 1.1 & 18.4 & $59.4 \%$ \\
B4 & 24 & 18.8 & 2.3 & 3.3 & 1.0 & 12.2 & $59.3 \%$ \\
B5 & 16 & 11.3 & 0.8 & 0.2 & 0.9 & 9.4 & $57.2 \%$ \\
B6 & 10 & 6.6 & 0.2 & 0.1 & 0.4 & 5.8 & $54.2 \%$ \\
& & \pm 0.6 & \pm 0.1 & \pm 0.1 & \pm 0.3 & \pm 0.5 & $\pm 0.8 \%$ \\
\hline
\end{tabular}

Table 2: The remaining numbers of events after each cut of the no missing energy selection for various background processes normalised to $614 \mathrm{pb}^{-1}$ are compared with data collected at $\sqrt{s}$ between $189 \mathrm{GeV}$ and $209 \mathrm{GeV}$. Efficiencies for a simulated event sample of $\mathrm{H}^{++} \mathrm{H}^{--}$with $\mathrm{H}^{ \pm \pm} \rightarrow \mathrm{e}^{ \pm} \mathrm{e}^{ \pm}$ and $M_{\mathrm{H}}=90 \mathrm{GeV} / c^{2}$ at $\sqrt{s}=206 \mathrm{GeV}$ are also given.

\begin{tabular}{|l||c|}
\hline Decay & efficiencies (\%) \\
\hline \hline $\mathrm{ee}$ & $42-61$ \\
$\mu \mu$ & $66-71$ \\
$\tau \tau$ & $42-46$ \\
$\mathrm{e} \mu$ & $55-65$ \\
$\mathrm{e} \tau$ & $33-40$ \\
$\mu \tau$ & $44-47$ \\
\hline
\end{tabular}

Table 3: Ranges of signal detection efficiencies (in \%) for the different $\mathrm{H}^{ \pm \pm}$masses (between $45 \mathrm{GeV} / c^{2}$ and $102 \mathrm{GeV} / c^{2}$ ) for the various decays. 


\begin{tabular}{|l||c|c|}
\hline \multirow{2}{*}{ Decay } & $\mathrm{H}_{\mathrm{L}}^{ \pm \pm}$ & $\mathrm{H}_{\mathrm{R}}^{ \pm \pm}$ \\
\hline \hline ee & $100.2 \mathrm{GeV} / c^{2}$ & $99.9 \mathrm{GeV} / c^{2}$ \\
$\mu \mu$ & $100.5 \mathrm{GeV} / c^{2}$ & $100.1 \mathrm{GeV} / c^{2}$ \\
$\tau \tau$ & $99.0 \mathrm{GeV} / c^{2}$ & $98.5 \mathrm{GeV} / c^{2}$ \\
$\mathrm{e} \mu$ & $100.4 \mathrm{GeV} / c^{2}$ & $100.0 \mathrm{GeV} / c^{2}$ \\
$\mathrm{e} \tau$ & $99.3 \mathrm{GeV} / c^{2}$ & $98.7 \mathrm{GeV} / c^{2}$ \\
$\mu \tau$ & $99.6 \mathrm{GeV} / c^{2}$ & $99.3 \mathrm{GeV} / c^{2}$ \\
\hline
\end{tabular}

Table 4: Mass limits at the $95 \%$ confidence level for left-handed and right-handed doubly charged Higgs bosons $\mathrm{H}^{ \pm \pm}$. 


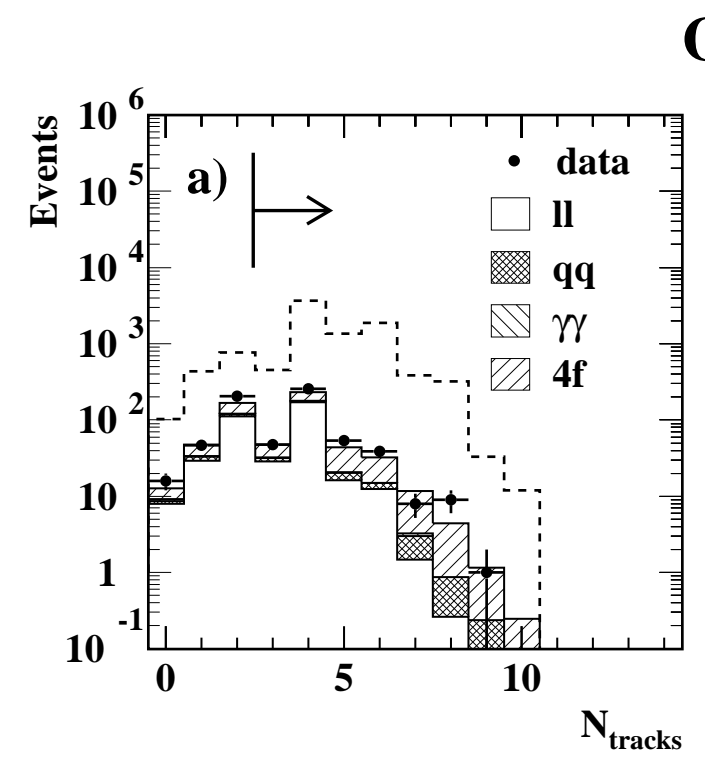

OPAL
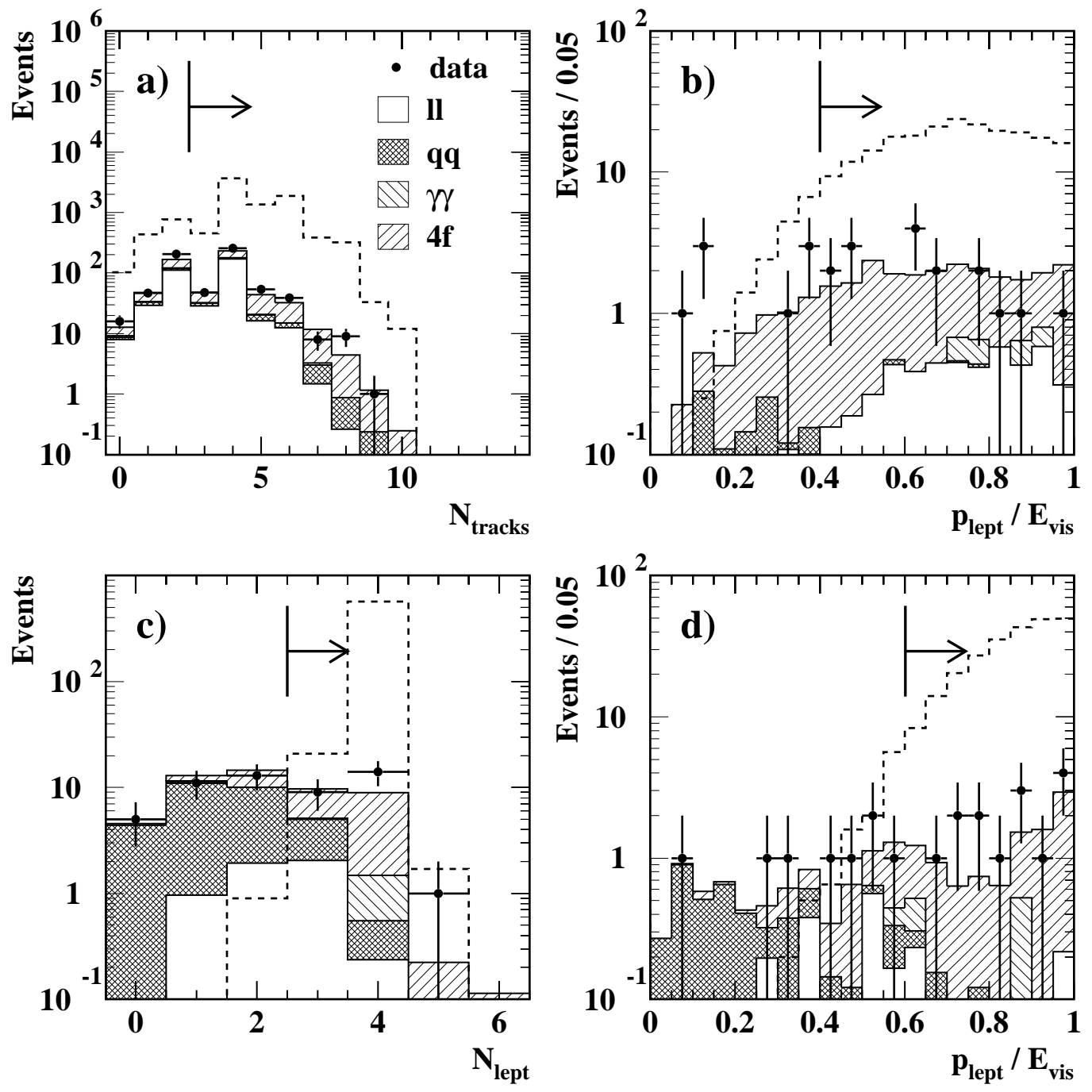

Figure 1: Distributions of some essential observables used in the selections: a) The number of tracks with transverse momentum larger than $1.5 \mathrm{GeV} / c, N_{\text {tracks }}$, after cut (A2) in the missing energy selection. b) The total leptonic momentum divided by the total visible energy of the event, $p_{\text {lept }} / E_{\text {vis }}$, after cut (A4) in the missing energy selection. c) The number of well identified isolated leptons, $N_{\text {lept }}$, after cut (B3) in the no missing energy selection. d) $p_{\text {lept }} / E_{\text {vis }}$ after cut (B4) in the no missing energy selection. The data are shown with error bars and distributions from the background processes are shown as solid line histograms: dilepton events (open area), multihadronic events (double hatched area), two-photon processes (negative slope hatching area), and four-fermion processes (positive slope hatching area). The arrows, pointing into the accepted regions, show where the analysis cuts are applied. The dashed line histograms show the predictions for a doubly-charged Higgs boson signal with $M_{\mathrm{H}}=90 \mathrm{GeV} / \mathrm{c}^{2}$ at $\sqrt{s}=206 \mathrm{GeV}$ with $100 \%$ decay into $\tau \tau$ for a) and b) and $100 \%$ decay into ee for c) and d). The normalisations of the signal histograms are arbitrary. 


\section{OPAL}
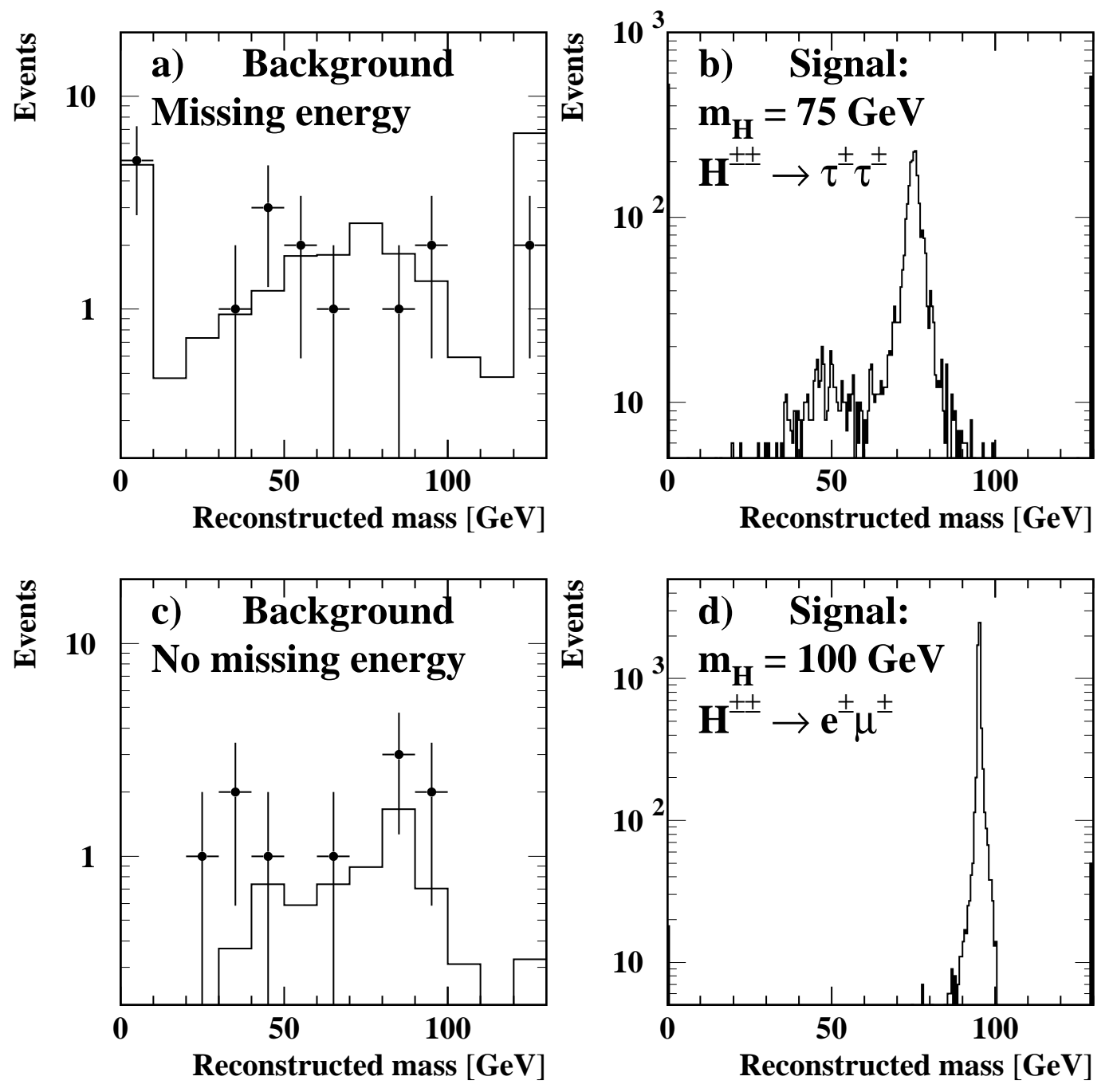

Figure 2: Distributions of the reconstructed Higgs boson mass for a) background (solid-line histogram) and data (points with error bars) and b) a signal with $M_{\mathrm{H}}=75 \mathrm{GeV} / \mathrm{c}^{2}$ with $100 \%$ decay into $\tau \tau$ at $\sqrt{s}=206 \mathrm{GeV}$, in the missing energy selection, and c) background (solid-line histogram) and data (points with error bars) and d) a signal with $M_{\mathrm{H}}=100 \mathrm{GeV} / \mathrm{c}^{2}$ with $100 \%$ decay into ee, at $\sqrt{s}=206 \mathrm{GeV}$, in the no missing energy selection. 


\section{OPAL}
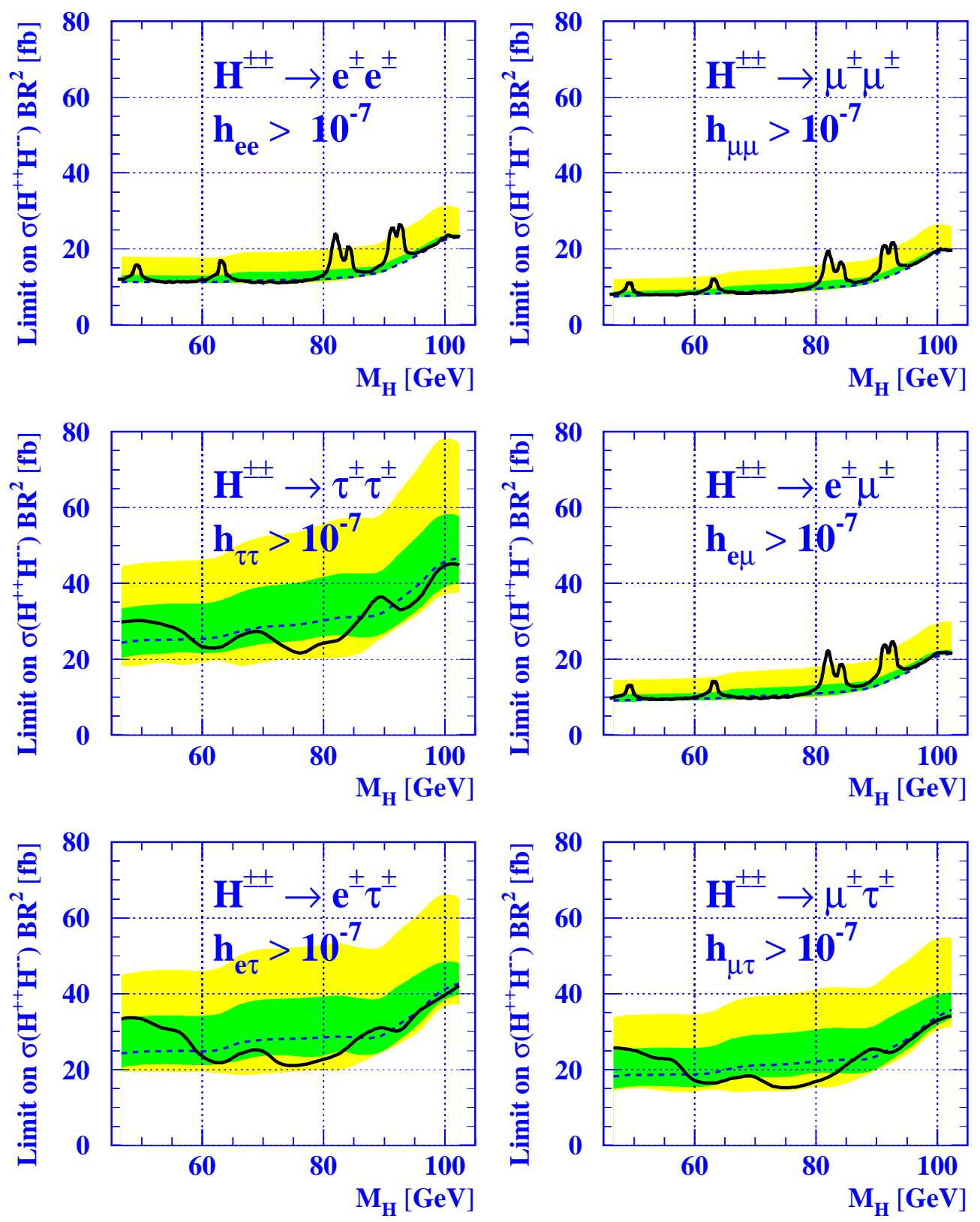

Figure 3: The 95\% confidence level upper limits on $\sigma\left(\mathrm{e}^{+} \mathrm{e}^{-} \rightarrow \mathrm{H}^{++} \mathrm{H}^{--}\right) \times B R^{2}\left(\mathrm{H}^{ \pm \pm} \rightarrow \ell^{ \pm} \ell^{ \pm}\right)$for each of the six possible leptonic decays at $\sqrt{s}=206 \mathrm{GeV}$. On each plot, the solid line shows the observed limit, while the dotted line shows the limit expected for background only. The dark/light shaded bands around the background expectation represent the $\pm 1 / \pm 2$ standard deviation spread. 


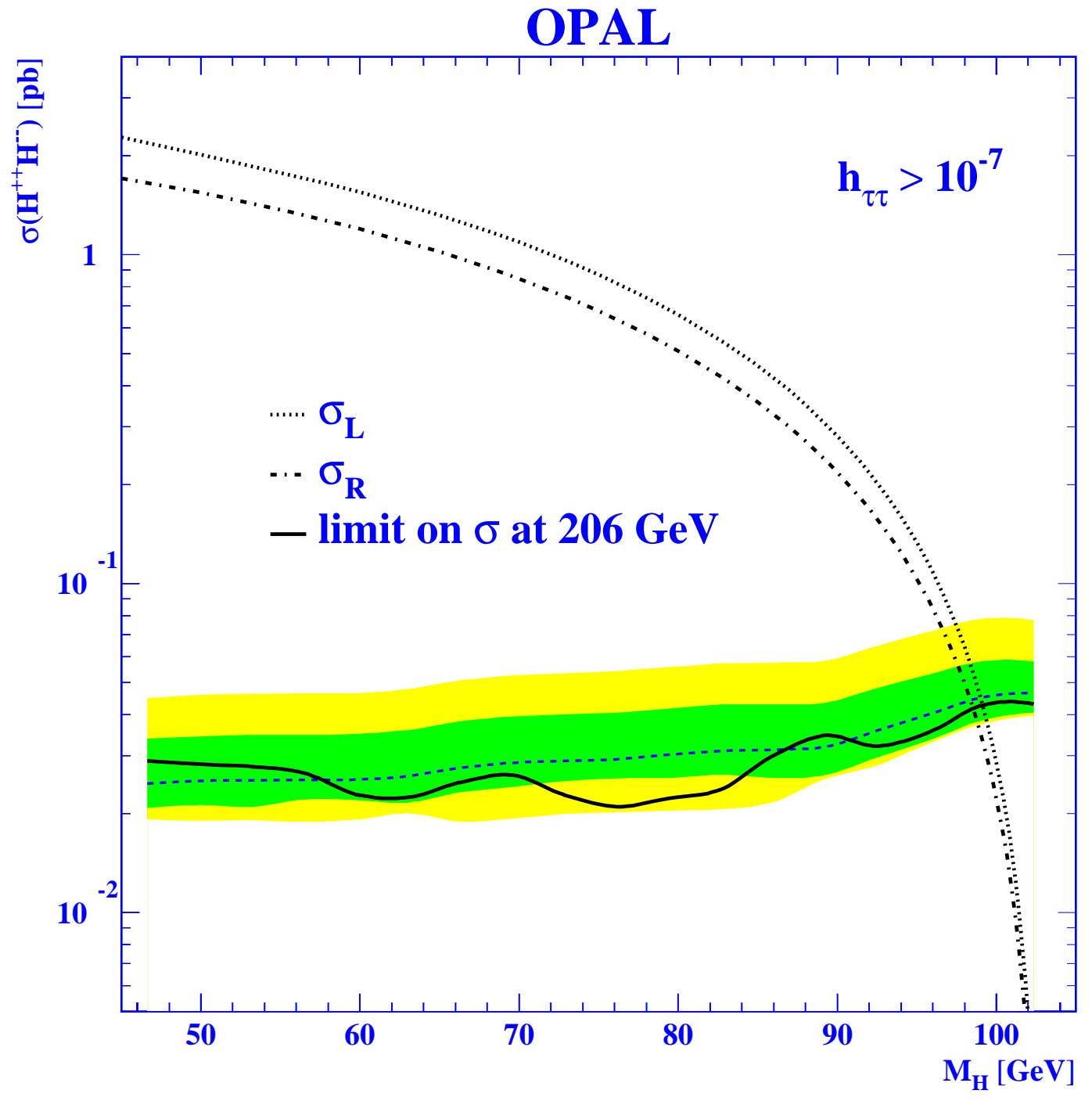

Figure 4: The solid line shows the 95\% confidence level upper limit on the $\mathrm{H}^{ \pm \pm}$pair production cross-section at $\sqrt{s}=206 \mathrm{GeV}$ assuming $100 \%$ branching ratio for the decay of $\mathrm{H}^{ \pm \pm}$into $\tau^{ \pm} \tau^{ \pm}$. The dashed line shows the limit expected for background only. The dark/light shaded bands around the background expectation represent the $\pm 1 / \pm 2$ standard deviation spread. The dotted and dashdotted lines show the expected production cross-sections of $\mathrm{H}_{\mathrm{L}}^{++} \mathrm{H}_{\mathrm{L}}^{--}$and $\mathrm{H}_{\mathrm{R}}^{++} \mathrm{H}_{\mathrm{R}}^{--}$in left-right symmetric models. All data collected at $\sqrt{s}$ between $189 \mathrm{GeV}$ and $209 \mathrm{GeV}$ have been combined and the production cross-section $\sigma$ has been assumed to vary linearly with $\beta^{3} / s$. 


\section{OPAL}
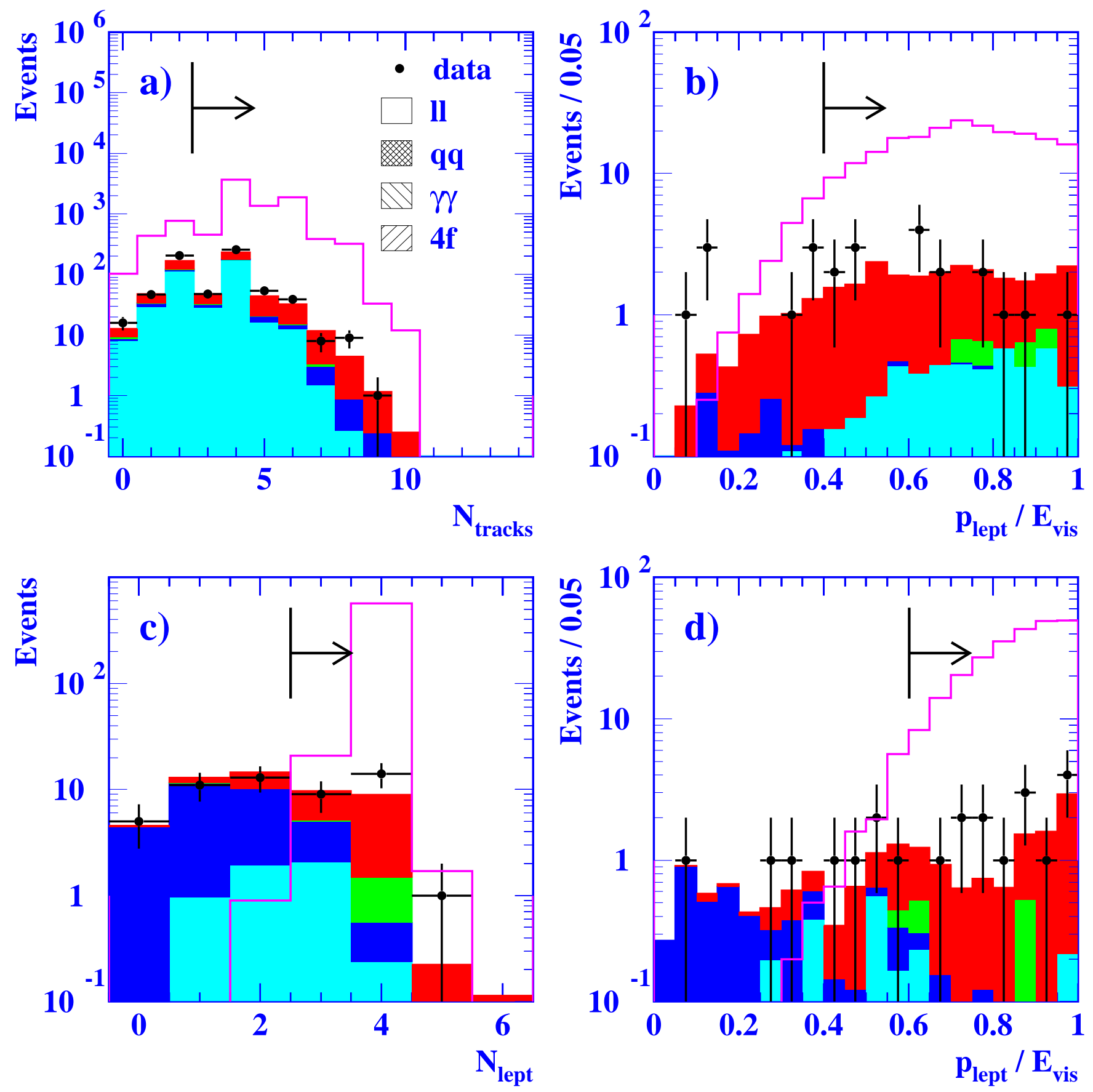
